\title{
CHARACTERISTIC IDEALS AND THE STRUCTURE OF LIE ALGEBRAS
}

\author{
GEORGE B. SELIGMAN
}

The purpose of this note is to study the radical and such concepts as semi-simplicity and simplicity for Lie algebras in terms of characteristic ideals. The program follows the outlines of part of a fundamental paper of Fitting on finite groups, and the final result displaying an isomorphism of an algebra $\mathfrak{R}$ with no solvable characteristic ideals into the Lie algebra of derivations of a uniquely determined completely reducible characteristic ideal in $\mathbb{R}$ is an exact analogue of a result of his on semi-simple groups. In case the base field is of characteristic zero, the theory collapses to the classical theory. An example is given to show that this is not the case at prime characteristic.

1. Preliminaries. Let $\mathfrak{R}$ be a finite-dimensional Lie algebra over a field $\mathfrak{F}$. A derivation $D$ of $\mathfrak{R}$ is a linear mapping of $\mathfrak{R}$ into itself such that $[x y] D=[x D, y]+[x, y D]$ for all $x, y \in \mathbb{R}$. In particular, the mapping ad $z: x \rightarrow[x z]$ is a derivation of $\&$ for every $z \in \mathbb{R}$. An ideal $\Im$ in $\mathfrak{R}$ is called a characteristic ideal in $\mathfrak{R}$ if $\Im D \subseteq \Im$ for every derivation $D$ of $\&$.

Let $\mathfrak{R}^{(1)}=\mathfrak{R}, \mathfrak{R}^{(2)}=[\mathbb{R}]$ (i.e., the space of all finite sums $\sum\left[x_{i} y_{i}\right]$, $\left.x_{i}, y_{i} \in \mathfrak{R}\right), \cdots, \mathfrak{R}^{(i+1)}=\left[\mathbb{R}^{(i)} \mathbb{R}^{(i)}\right], \cdots$ Then each $\mathfrak{R}^{(i)}$ is a characteristic ideal in $\mathfrak{\ell}$, and $\ell$ is called solvable if some $\mathfrak{R}^{(i)}=(0)$. If $\Im_{\text {is a }}$ solvable ideal in $\mathscr{R}$, and if the quotient algebra $\mathscr{R} / \Im$ is solvable, then $\mathfrak{R}$ is solvable. For the solvability of $\mathfrak{R} / \Im$ means that $\mathfrak{R}^{(i)} \subseteq \Im$ for some $i$. Then $\Re^{(i+\nu)} \subseteq \Im^{(\nu+1)}=0$ for suitable $\nu$. Now if $\Re$ and $\subseteq$ are solvable ideals in $\mathbb{R}$, so is $\Re+\subseteq$ (set of all sums $r+s, r \in \Re, s \in \subseteq$ ). For by the fundamental isomorphism theorem, $\Re+\mathfrak{S} / \mathfrak{S} \cong \Re / \Re \cap \subseteq$ is solvable; since $\subseteq$ is also solvable, so is $\Re+\subseteq$. It is clear that $\Re+\subseteq$ is an ideal and is in fact a characteristic ideal if both $\Re$ and $\subseteq$ are characteristic ideals. Consequently the sum of all solvable characteristic ideals in a Lie algebra $\mathfrak{R}$ is again a solvable characteristic ideal, and is in fact the only maximal solvable characteristic ideal in $\&$. We shall call this ideal the characteristic radical, or c-radical, of $\mathbb{R}$.

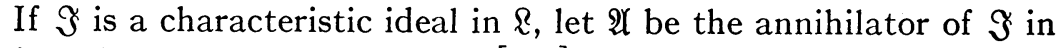
$\mathfrak{R}$, i.e., the set of $x \in \mathfrak{R}$ such that $[x \mathfrak{\Im}]=(0)$. Then $\mathfrak{A}$ is clearly a subspace of $\mathfrak{R}$; in fact, $\mathfrak{A}$ is a characteristic ideal in $\mathfrak{R}$. For if $D$ is a derivation of $\mathfrak{R}$, then 1956.

Presented to the Society, April 21, 1956; received by the editors March 26, 


$$
[\mathfrak{H} D, \Im] \subseteq[\mathfrak{A} \Im] D+[\mathfrak{A}, \Im D] \subseteq[\mathfrak{A} \Im]=(0),
$$

and this is sufficient to show that $\mathfrak{A}$ is an ideal (take $D=\operatorname{ad} y, y \in \mathfrak{R}$ ).

2. Characteristic semi-simple algebras. A Lie algebra $\&$ will be called characteristic semi-simple (css.) if its $c$-radical is (0), or equivalently, if its only solvable characteristic ideal is $(0)$. By considering the derived sequence $\mathfrak{A}^{(i)}$ of a solvable ideal $\mathfrak{A}$, we see from the last nonzero entry that $\mathfrak{R}$ is css. if and only if the only abelian characteristic ideal is $(0)$. (A Lie algebra $\mathfrak{R}$ is abelian if $[R \mathbb{R}]=(0)$.)

Theorem 1. If $\Re$ is the c-radical of $\mathbb{R}$, then $\mathbb{R} / \Re$ is css.

Proof. Let $\mathfrak{S}$ be a solvable characteristic ideal of $\mathfrak{R} / \Re$. Let $\phi$ denote the natural homomorphism of $\mathfrak{R}$ onto $\mathfrak{R} / \mathfrak{R}$. Let $\Im^{*}=\subseteq \phi^{-1}$. Then $\mathfrak{S}^{*}$ is a solvable ideal of $\mathbb{R}$, since some entry in the sequence of $\left(\mathfrak{S}^{*}\right)^{(i)}$ is contained in $\Re$. Let $D$ be a derivation of $R$. Then $D$ maps $\Re$ into itself, hence induces a derivation $\bar{D}$ of $\Re / \Re$, defined by

$$
(u \phi) \bar{D}=(u D) \phi, \quad u \in \Omega .
$$

In particular, $\subseteq \bar{D} \subseteq \subseteq$, since $\mathfrak{S}$ is a characteristic ideal in $\mathfrak{R} / \Re$. Now let $s \in \mathfrak{S}^{*}$. Then $s \phi \in \mathfrak{S}$, and $(s D) \phi=(s \phi) \bar{D} \in \mathfrak{S}$, or $s D \in \mathfrak{S}^{*}$. Thus $\mathfrak{S}^{*}$ is a characteristic ideal in $\mathfrak{R}$ (to prove a subspace is a characteristic ideal, it suffices to prove that it is mapped into itself by all derivations $D$; the ideal property followed by taking $D=$ ad $x, x \in \mathfrak{R})$. Consequently, since $\mathfrak{S}^{*} \supseteq \Re$, we have $\mathfrak{S}^{*}=\Re$, or $\mathfrak{S}=(0)$. This completes the proof.

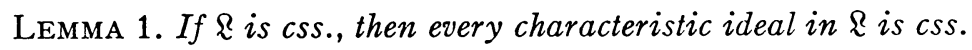

Proof. Let $\mathbb{R}$ be css., $\mathfrak{A}$ a characteristic ideal in $\mathfrak{R}$. Let $\mathbb{S}$ be a solvable characteristic ideal in $\mathfrak{A}$. Let $D$ be a derivation of $\mathfrak{R}$. Since $\mathfrak{A} D \subseteq \mathfrak{A}$, the restriction of $D$ to $\mathfrak{A}$ is a derivation of $\mathfrak{A}$, therefore maps

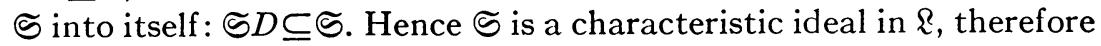
is $(0)$.

A Lie algebra $\&$ is called characteristic simple (c-simple) if its only characteristic ideals are $\mathfrak{R}$ and $(0)$, and if $[R \mathbb{R}]=\mathfrak{\ell}$. We can say something about the ideals in such an algebra, although the concept is somewhat looser than ordinary simplicity when the characteristic of the base field $\mathfrak{F}$ is not zero. Evidently a $c$-simple algebra is css.

Theorem 2. Every proper ideal in a characteristic simple algebra $\&$ is nilpotent.

Proof. Let $\mathfrak{A}$ be an ideal in $\mathfrak{R},(0) \neq \mathfrak{V} \neq \mathfrak{R}$. First observe that if $D$ is any derivation of $\mathfrak{R}$, 


$$
[\mathfrak{H} \mathfrak{A}] D \subseteq[\mathfrak{H} D, \mathfrak{A}]+[\mathfrak{U}, \mathfrak{A} D] \subseteq[\mathfrak{R}] \subseteq \mathfrak{A} .
$$

More generally, if $\mathfrak{A}^{k}=[\cdots[[\mathfrak{A} \mathfrak{A}] \mathfrak{A}] \cdots \mathfrak{A}]$ ( $k$ factors), and if $\mathfrak{H}^{k} D \subseteq \mathfrak{P}^{k-1}$, then

$$
\begin{aligned}
& \mathfrak{A}^{k+1} D=\left[\mathfrak{H}^{k \mathfrak{U}}\right] D \subseteq\left[\mathfrak{A}^{k} D, \mathfrak{U}\right]+\left[\mathfrak{H}^{k}, \mathfrak{A} D\right] \\
& \subseteq\left[\mathfrak{H}^{k-1}, \mathfrak{A}\right]+\left[\mathfrak{Q}^{k}, \mathfrak{l}\right] \subseteq \mathfrak{U}^{k} \text {. }
\end{aligned}
$$

Thus it follows by induction that $\mathfrak{A}^{n+1} D \subseteq \mathfrak{A}^{n}$ for all $n$.

Now the sequence $\mathfrak{A}^{n}$ is a descending chain of ideals, so we must have $\mathfrak{A}^{n+1}=\mathfrak{Y}^{n}$ for some $n$. Then $\mathfrak{A}^{n} D=\mathfrak{A}^{n+1} D \subseteq \mathfrak{H}^{n}$ for all derivations $D$ of $\mathscr{R}$, or $\mathfrak{U}^{n}$ is a characteristic ideal. Consequently $\mathfrak{U}^{n}=(0)$, or $\mathfrak{A}$ is nilpotent.

3. Completely semi-simple algebras. A Lie algebra $\&$ will be called completely semi-simple if $\mathbb{R}$ can be written as a direct sum of a set of ideals in $\mathfrak{R}$ which are characteristic simple algebras. Let $\mathfrak{R}$ be completely semi-simple, and let

$$
\mathbb{R}=\Omega_{1} \oplus \cdots \oplus \Omega_{s}
$$

be a direct decomposition of $\Omega$ as a sum of ideals which are $c$-simple algebras. Let us prove that the $\mathfrak{R}_{i}$ are characteristic ideals in $\mathfrak{R}$. To this end, we first show that $R_{2}+\cdots+\Omega_{s}$ is the annihilator of $\Omega_{1}$ in $\mathfrak{R}$, and vice versa. Since $\left[R_{i} R_{j}\right]=(0)$ if $i \neq j$, it is enough to show that the respective annihilators are contained in the corresponding ideals.

Let $x=x_{1}+x_{2}+\cdots+x_{s}, x_{i} \in \Omega_{i}$, be in the annihilator of $\Omega_{1}$. We prove $x_{1}=0$. If $x_{1} \neq 0$, then $\left[x_{1} R_{1}\right]=\left[x R_{1}\right]=(0)$. Let $\mathfrak{C}_{1}$ be the center of $\Omega_{1}$, i.e., the annihilator of $\ell_{1}$ in $\Re_{1}$. Then $\mathbb{S}_{1}$ is a characteristic ideal in $\Omega_{1}$. Since $\left[\mathfrak{R}_{1} \mathfrak{R}_{1}\right]=\mathfrak{R}_{1}, \mathfrak{C}_{1}=(0)$. It follows that $x_{1}=0$, or $x \in \mathfrak{R}_{2}+\cdots$ $+\mathfrak{R}_{s}$. If $x=x_{1}+\cdots+x_{s}$ is in the annihilator of $\Omega_{2}+\cdots+\Omega_{s}$, let $x_{j} \neq 0, j>1$. Then $x_{j}$ is in the center of $\mathfrak{E}_{j}$ of $\mathfrak{R}_{j}$, a contradiction. Therefore $x=x_{1} \in \Omega_{1}$, and the assertion about the annihilators is proved.

Now let $x_{1} \in \mathfrak{R}_{1}$, and let $D$ be a derivation of $\mathfrak{R}$. Let $y \in \mathfrak{R}_{2}+\cdots+\mathfrak{\Omega}_{s}$. Then

$$
\left[x_{1} D, y\right]=\left[x_{1} y\right] D-\left[x_{1}, y D\right]=-\left[x_{1}, y D\right] .
$$

Thus $\left[x_{1} D, y\right] \in\left(\mathfrak{R}_{2}+\cdots+\mathfrak{R}_{s}\right) \cap \mathfrak{R}_{1}=(0)$, or $x_{1} D$ is in the annihilator of $\ell_{2}+\cdots+\ell_{s}$, i.e., in $\Omega_{1}$. Therefore $\mathfrak{R}_{1}$ is a characteristic ideal in $\mathfrak{R}$, and the same holds for each $\mathfrak{R}_{i}$.

Let $\&$ be completely semi-simple, and let $\Im$ be a characteristic ideal in $\mathfrak{R}$. If $\mathfrak{R}=\mathfrak{R}_{1} \oplus \cdots \oplus \mathfrak{R}_{s}, \mathfrak{R}_{i} c$-simple ideals, then $\left[\mathfrak{S}_{i}\right]$ is a character-

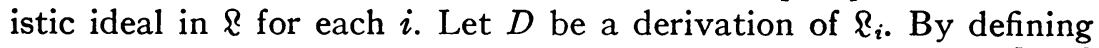
$\ell_{j} D=(0), j \neq i$, we can extend $D$ to a derivation of $\ell_{\text {. Therefore }}\left[\Im_{i}\right]$ is a characteristic ideal in $R_{i}$, and is either $R_{i}$ or $(0)$. If $\left[\Im R_{i}\right]=(0)$, 
then $\Im \subseteq R_{1}+\cdots+R_{i-1}+R_{i+1}+\cdots+R_{s}$, the annihilator of $R_{i}$. If $\left[\Im \Omega_{i}\right]=\Omega_{i}$, then $\Omega_{i} \subseteq \Im$. It follows that $\Im$ is the (direct) sum of those $\mathfrak{R}_{i}$ such that $\left[\mathfrak{R}_{i}\right]=\mathfrak{R}_{i}$, and in particular that $[\Im \Im]=\Im$. We can therefore conclude:

TheOREM 3. Every completely ss. Lie algebra is css.

\section{The maximal completely semi-simple characteristic ideal.}

THEOREM 4. Let $\mathfrak{R}$ be css. Then $\mathfrak{R}$ contains a nonzero completely semi-simple characteristic ideal.

Proof. We proceed by induction on the dimension of $\mathfrak{R}$. If $\mathfrak{R}$ has the smallest possible dimension among all css. algebras (namely, 3), then $\&$ must be characteristic simple, since by Lemma 1 every characteristic ideal in $\mathbb{R}$ in css. In this case the theorem is trivial. Now assume the theorem has been proved for css. algebras of dimensions less than $k$, and let $\&$ have dimension $k$. If $\&$ is $c$-simple, then $\&$ is completely ss. If not, $\mathbb{R}$ contains a proper characteristic ideal $\mathfrak{A}$, which in turn is css. Since $\mathfrak{A}$ is of dimension less than $k, \mathfrak{A}$ contains a nonzero completely ss. characteristic ideal $\mathfrak{S}$. But if $D$ is a derivation of $\mathfrak{R}$, then $\mathfrak{A} D \subseteq \mathfrak{A}$, so $\mathfrak{S} D \subseteq \subseteq$, i.e., $\mathfrak{S}$ is a characteristic ideal in $\mathfrak{R}$. This completes the proof.

LEMMA 2. If $\&$ is css., the sum of any two completely semi-simple characteristic ideals in $\mathbb{R}$ is completely semi-simple.

Proof. Let $\Im=\Im_{1} \oplus \cdots \oplus \Im_{r}$ and $\Re=\Omega_{1} \oplus \cdots \oplus \Omega_{8}$ be completely semi-simple characteristic ideals in $\&$; the $\Im_{i}$ are $c$-simple ideals in $\Im$, the $\Omega_{i} c$-simple ideals in $\Omega$. Then

$$
\Im+\Omega=\Im_{1}+\cdots+\Im_{r}+\Omega_{1}+\cdots+\Omega_{s} .
$$

All $\Im_{i}$ and $\Omega_{i}$ are characteristic ideals in $\mathfrak{R}$, since they are characteristic ideals in $\Im$ and $\Omega$, respectively. Now consider

$$
\left[\Im_{1}+\cdots+\Im_{r}+\Omega_{1}+\cdots+\Re_{s-1}, \Omega_{s}\right]=\mathfrak{A} .
$$

This is a characteristic ideal in $\mathfrak{R}$ contained in $\Omega_{s}$, and is an ideal in $\Omega_{8}$. Since $\Omega_{s}$ is $c$-simple, we have by Theorem 2 , that either $\mathfrak{A}=\Omega_{s}$, $\mathfrak{A}=(0)$, or $\mathfrak{A}$ is nilpotent. But in the last of these cases, $\mathfrak{A}$ is a nilpotent (hence solvable) characteristic ideal in $\mathfrak{R}$, therefore must be (0). Since $\mathfrak{A} \subseteq \Im_{1}+\cdots+\Re_{s-1}$, we have

$$
\Re_{8} \subseteq \Im_{1}+\cdots+\Re_{8-1} \text { in case } \mathfrak{A}=\Omega_{8} \text {, and otherwise }
$$

$\left(\Im_{1}+\cdots+\Omega_{s-1}\right) \cap \Omega_{s}$ is in the center of $\Omega_{s}$, hence is (0).

In this case, $\Im+\Omega=\left(\Im_{1}+\cdots+\Omega_{8-1}\right) \oplus \Omega_{8}$. We can now repeat the 
argument using $\Im_{1}+\cdots+\Omega_{s-1}$ to prove that $\Im+\Omega$ is a direct sum of certain of the $\Im_{i}$ and certain of the $\Omega_{j}$. This completes the proof of the lemma.

It follows from Lemma 2 and Theorem 4 that every css. algebra $\&$ contains a unique nonzero maximal completely ss. characteristic ideal $\mathfrak{S}$. Let $\mathfrak{A}$ be the annihilator of $\mathfrak{S}$ in $\mathfrak{R} ; \mathfrak{A}$ is a characteristic ideal in $\mathfrak{R}$. Therefore $\mathfrak{A} \cap \mathfrak{S}$ is an abelian characteristic ideal in $\mathfrak{R}$, hence is $(0)$, and $\mathfrak{A}+\mathfrak{S}=\mathfrak{A} \oplus \mathfrak{S}$. If $\mathfrak{A} \neq(0), \mathfrak{A}$ contains a nonzero completely ss. characteristic ideal $\mathfrak{S}_{1}$, which is also a characteristic ideal in $\mathbb{R}$. Therefore $\mathfrak{S}_{1}+\mathfrak{S}=\mathfrak{S}_{1} \oplus \mathfrak{S}$ is a completely ss. ideal in $\mathfrak{R}$ containing $\mathfrak{S}$ properly. This is a contradiction. If we denote by ads $(x)$ the restriction to the invariant subspace $\mathfrak{S}$ of the mapping ad $(x), x \in \mathbb{R}$, we have the following theorem.

THEOREM 5. The annihilator of $\mathbb{S}$ in $\mathbb{R}$ is (0). Therefore the mapping $x \rightarrow \operatorname{ad} \Subset(x)$ is an isomorphism of $\mathbb{R}$ into the derivation algebra $\mathfrak{D}$ of $\mathfrak{S}$.

It will be observed that the image of $\mathbb{R}$ under this isomorphism contains in particular all inner derivations of $\mathfrak{S}$, and that if every derivation of $\mathfrak{S}$ should be inner, we would have $\mathfrak{l}=\mathfrak{S}$. Moreover, $\operatorname{ad} \mathfrak{S}(\mathfrak{R})$ is an ideal in $\mathfrak{D}$ if and only if every derivation of $\mathfrak{S}$ can be extended to a derivation of $\mathbb{R}$. For if this condition is satisfied, let $D$ be a derivation of $\mathfrak{S}$, and let $x \in \mathfrak{R}$. We denote also by $D$ some extension of $D$ to a derivation of $R$. If $s \in \mathcal{S}$,

$$
s[\operatorname{ads}(x), D]=[s, x] D-[s D, x]=[s, x D],
$$

or

$$
[\operatorname{ad} s(x), D]=\operatorname{ad} \varsigma(x D) \in \operatorname{ad}((\mathbb{R}) .
$$

Conversely if $\operatorname{ad} \Subset(\mathfrak{R})$ is an ideal in $\mathfrak{D}$, let $D \in \mathfrak{D}$. Then for every $x \in \mathbb{R}$, there exists $y \in \mathbb{R}$ such that $[\operatorname{ad} \subseteq(x), D]=\operatorname{ad} \subseteq(y)$. (In fact, $y$ is unique by Theorem 5.) Define $x D=y$. Then $D$ is evidently a linear mapping of $\mathfrak{R}$ into $R$, and if $x_{1}, x_{2} \in \mathbb{R}$,

$$
\begin{aligned}
& \operatorname{ad}\left(\left[x_{1} x_{2}\right] D\right)=\left[\operatorname{ad} \Phi\left(\left[x_{1} x_{2}\right]\right), D\right]=\left[\left[\operatorname{ad}\left(x_{1}\right), \operatorname{ad}\left(x_{2}\right)\right] D\right] \\
& =-\left[\left[\operatorname{ad}\left(x_{2}\right), D\right] \operatorname{ad} \varsigma\left(x_{1}\right)\right]-\left[\left[D, \operatorname{ad} \varsigma\left(x_{1}\right)\right] \operatorname{ad} \Phi\left(x_{2}\right)\right] \\
& =-\left[\operatorname{ad} \varsigma\left(x_{2} D\right), \operatorname{ad} \varsigma\left(x_{1}\right)\right]+\left[\operatorname{ad} \varsigma\left(x_{1} D\right), \operatorname{ad} \subseteq\left(x_{2}\right)\right] \\
& =\operatorname{ads}\left(\left[x_{1} D, x_{2}\right]+\left[x_{1}, x_{2} D\right]\right) \text {, }
\end{aligned}
$$

so that $D$ is a derivation of $\&$ coinciding with the given derivation on $\mathfrak{S}$.

5. An example. The following example, suggested to me by Professor Jacobson, will illustrate some points where the theory exposed 
here diverges from the standard notions of radical and simplicity. Let $\mathfrak{F}$ be a field of characteristic $p>2$ and let $\mathfrak{M}$ be the three-dimensional simple Lie algebra of two by two matrices of trace zero over $\mathfrak{F}$. Let (S) be a cyclic group of order $p$ with generator $g$, and let $\mathfrak{A}$ be the group ring of $\mathfrak{F}$ over $\mathfrak{F}$. Let the space $\mathfrak{R}$ be defined as the tensor product $\mathfrak{M} \otimes \mathfrak{A}$. We can make $\mathfrak{R}$ into a Lie algebra by defining $\left[m_{1} \otimes a_{1}\right.$, $\left.m_{2} \otimes a_{2}\right]=\left[m_{1} m_{2}\right] \otimes a_{1} a_{2}$. Among the derivations of $\&$ we have, in addition to the inner derivations, all mappings of the form $m \otimes a \rightarrow m$ $\otimes a^{\prime}$, where $a \rightarrow a^{\prime}$ is a derivation of $\mathfrak{A}$, as an associative algebra. Now the associative radical $\mathfrak{R}$ of $\mathfrak{A}$ is spanned by $g-1, g^{2}-1, \cdots, g^{p-1}-1$, and $\mathfrak{M} \otimes \mathfrak{N}$ is a nonzero solvable ideal in $\mathfrak{R}$, in fact a nilpotent ideal. However the mapping $g \rightarrow 1$ defines a derivation of $\mathfrak{A}$, which induces a derivation $D$ of $\mathfrak{R}$ as above. If $\mathfrak{B}$ is a characteristic ideal in $\mathfrak{R}, \mathfrak{B} \neq(0)$, then one operates successively with $D$ and with the derivations ad $(m \otimes 1), m \in \mathfrak{M}$, to show $\mathfrak{B}$ contains the elements $m_{1} \otimes 1, m_{2} \otimes 1$, $m_{3} \otimes 1$, where $m_{1}, m_{2}, m_{3}$ are a basis for $\mathfrak{M}$. Then operation with the derivations ad $\left(m_{\boldsymbol{i}} \otimes g^{j}\right)$ shows that $\mathfrak{B}=\mathfrak{R}$. Therefore $\mathfrak{R}$ is $c$-simple, but not simple, and its radical is not a characteristic ideal.

\section{BIBLIOGRAPHY}

1. H. Fitting, Beiträge zur Theorie der Gruppen endlicher Ordnung, Jber. Deutschen Math. Verein. vol. 48 (1938) pp. 77-141.

\section{Princeton University}

